Journal for ImmunoTherapy of Cancer

\title{
Role of myeloid-derived suppressor cells in the promotion and immunotherapy of colitis- associated cancer
}

\author{
Yungang Wang, ${ }^{1}$ Yanxia Ding, ${ }^{2}$ Yijun Deng, ${ }^{3}$ Yu Zheng, ${ }^{1}$ Shengjun Wang (D) ${ }^{4}$
}

To cite: Wang $Y$, Ding $Y$, Deng $\mathrm{Y}$, et al. Role of myeloidderived suppressor cells in the promotion and immunotherapy of colitis-associated cancer. Journal for ImmunoTherapy of Cancer 2020;8:e000609. doi:10.1136/jitc-2020-000609

YW and YaD contributed equally.

Accepted 26 August 2020

\section{ABSTRACT}

Colitis-associated cancer (CAC) is a specific type of colorectal cancer that develops from inflammatory bowel disease (IBD). Myeloid-derived suppressor cells (MDSCs) are a heterogeneous population of immature myeloid cells that are essential for the pathological processes of inflammation and cancer. Accumulating evidence indicates that MDSCs play different but vital roles during IBD and CAC development and impede CAC immunotherapy. New insights into the regulatory network of MDSCs in the CAC pathogenesis are opening new avenues for developing strategies to enhance the effectiveness of CAC treatment. In this review, we explore the role of MDSCs in chronic inflammation, dysplasia and CAC and summarize the potential CAC therapeutic strategies based on MDSC blockade.

\section{INTRODUCTION}

Colitis-associated cancer (CAC) follows the sequence of normal tissue, hyperplasia, high-grade dysplasia, and adenocarcinoma. The etiology of CAC includes epigenetic changes, somatic mutations, and chronic inflammation. Inflammatory bowel disease (IBD), which is composed of Crohn's disease (CD) and ulcerative colitis (UC), is the result of continuous microbial antigen-induced immune responses as a consequence of host genetic defects in intestinal mucosal barrier function, immunomodulation, or bacterial killing. IBD is a high-risk factor for CAC. A meta-analysis of 54478 patients with UC, including 1698 cases of CAC, found that the overall prevalence of colorectal cancer (CRC) among UC cases was 3.7\%. ${ }^{2}$ The cumulative risk for CAC in patients with $\mathrm{CD}$ has been reported to be $18.4 \%$ after 30 years of disease duration. ${ }^{2}$ On the one hand, inflammation causes strong genotoxic reactions, such as DNA damage and mutations in important genes, which subsequently drive CAC initiation. On the other hand, inflammation activates the $W n t / \beta$-catenin signaling pathway, which induces intestinal epithelial cell (IEC) proliferation and remodeling and then promotes CAC development. CAC provides a great model to understand the role of chronic inflammation in tumors. However, the exact cause of chronic inflammation in patients with IBD and the key driver of the conversion from IBD to CAC still remain unknown.

The massive infiltration of myeloid cells and lymphocytes into the inflamed intestinal tissue is the main pathological feature of IBD. Dendritic cells (DCs) and macrophages sense invading micro-organisms and regulate the differentiation of proinflammatory lymphocytes such as T helper (Th1) cells, Th17 cells, innate lymphoid cells (ILCs), and interleukin (IL) $-17^{+} \gamma \delta \mathrm{T}$ cells. The lymphocytes further recruit myeloid cells into the local intestinal tissue. These myeloid cells play an important role in promoting the conversion of IBD to CAC. ${ }^{3}$ Myeloid-derived suppressor cells (MDSCs) are a heterogeneous population of myeloid progenitors and immature myeloid cells. In IBD and CAC, MDSCs massively infiltrate the inflamed intestinal tissue and tumor microenvironment and have been a research focus due to their roles in inflammation and tumorigenesis. ${ }^{4}$ Currently, the functional and phenotypic heterogeneity of MDSCs leads to controversy about their role in IBD. Furthermore, the mechanism by which MDSCs regulate the conversion from IBD to CAC is largely unknown. This review mainly focuses on the identification of MDSCs in patients with IBD or CAC, emerging insights into the regulatory network of MDSCs in IBD and CAC pathogenesis, and new possible avenues for CAC immunotherapy by targeting MDSCs.

\section{IDENTIFICATION OF MDSCS IN IBD/CAC}

MDSCs mainly include granulocytic and monocytic subsets. The identification of MDSC subsets in CAC is still difficult, which 
is mainly attributed to the diverse origins, heterogeneous phenotypes, and mutual transformation of myeloid cells.

\section{Identification of colorectal-infiltrating MDSCs}

The morphology and phenotype of granulocytic MDSCs (G-MDSCs or polymorphonuclear (PMN)-MDSCs) and monocytic MDSCs (M-MDSCs) are similar to those of neutrophils and monocytes, respectively. Murine MDSCs are defined as $\mathrm{Gr}-1^{+} \mathrm{CD} 11 \mathrm{~b}^{+}$cells. G-MDSCs and M-MDSCs are defined as $\mathrm{CD} 11 \mathrm{~b}^{+} \mathrm{Ly}_{6 \mathrm{G}} \mathrm{Ly}^{+} \mathrm{C}^{\mathrm{lo}}$

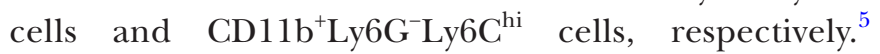
Human MDSCs are extensively described as HLA$\mathrm{DR}^{-} \mathrm{CD} 11 \mathrm{~b}^{+} \mathrm{CD} 33^{+}$cells. G-MDSCs and M-MDSCs are described as HLA-DR ${ }^{-} \mathrm{CD} 11 \mathrm{~b}^{+} \mathrm{CD} 33^{+} \mathrm{CD} 15^{+}$cells and HLA-DR ${ }^{-} \mathrm{CD} 11 \mathrm{~b}^{+} \mathrm{CD} 33^{+} \mathrm{CD} 14^{+} \quad$ cells, respectively. ${ }^{6}$ Intestine-infiltrating MDSCs were first described as $\mathrm{CD} 11 \mathrm{~b}^{+} \mathrm{Gr}-1^{+}$cells with immunosuppressive functions

A

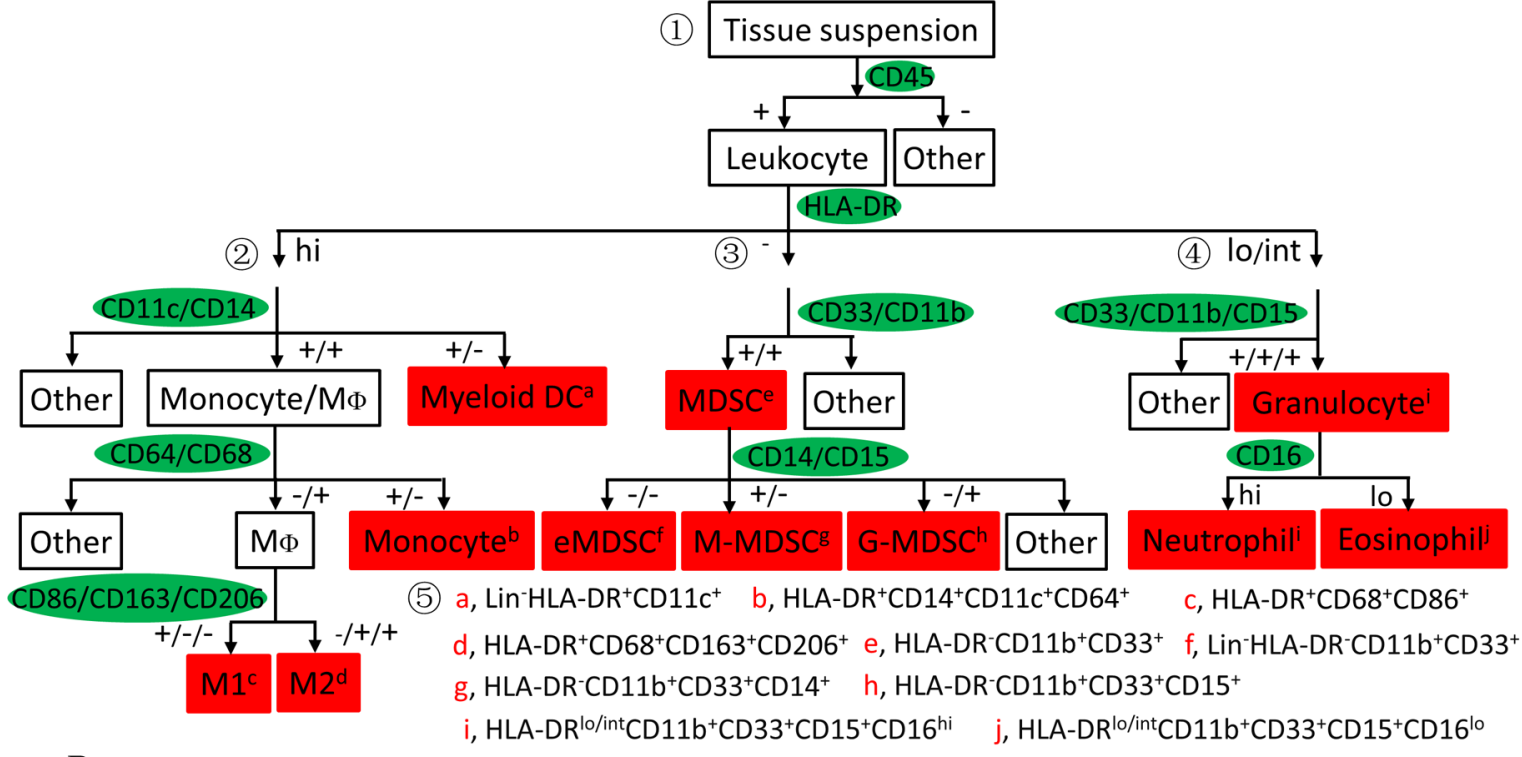

B

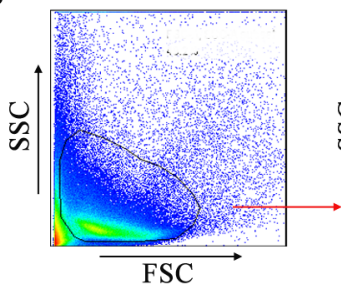

Isotype

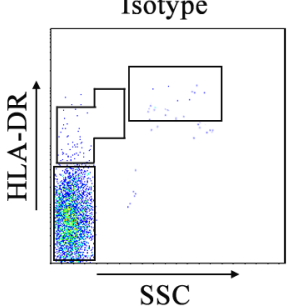

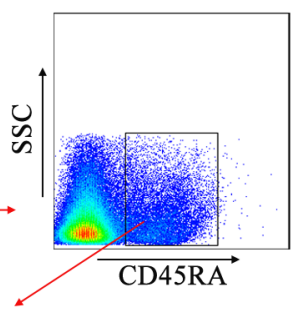

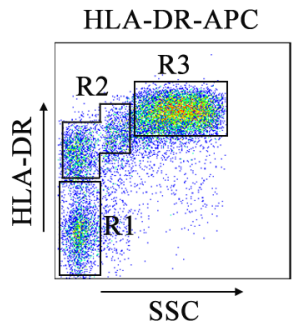

$\mathrm{C}$

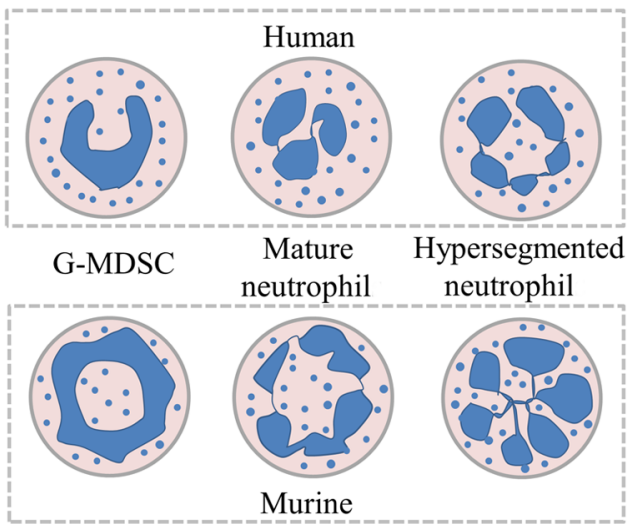

Figure 1 Strategy for the identification of human colorectal-infiltrating MDSCs. (A) Gating strategy of colorectal-infiltrating myeloid cells. (1) Leukocytes are divided into three groups based on HLA-DR levels. (2) Monocytes/MФs and DCs are distinguished from HLA-DR ${ }^{\text {hi }}$ cells based on the expression of CD11C and CD14. M1 and M2 macrophages are distinguished based on the expression of CD86, CD163, and CD206. (3) MDSCs are distinguished from HLA-DR ${ }^{-}$cells based on the expression of CD33 and CD11b. eMDSCs, M-MDSCs and G-MDSCs are distinguished based on the expression of CD14 and CD15. (4) Granulocytes are distinguished from HLA-DR ${ }^{\text {lo/int }}$ cells based on the expression of CD33, CD11b, and CD15. Neutrophils and eosinophils are distinguished based on the expression of CD16. (5) Immunophenotypes of human colorectalinfiltrating myeloid cells. (B) Representative FACS plots of leukocytes based on HLA-DR levels. Leukocytes were isolated from the CRC tissue suspension by CD45RA and then divided into three groups based on HLA-DR levels. R1: HLA-DR ${ }^{-}$cells; R2: HLA-DR ${ }^{\text {lo/int }}$ cells; R3: HLA-DR ${ }^{\text {hi }}$ cells. (C) Schematic representations of the nuclear morphology of G-MDSCs and neutrophils. APC, allophycocyanin; DC, dendritic cell; eMDSC, early-stage myeloid-derived suppressor cell; FACS, flow cytometry; FSC, forward scatter; G-MDSC, granulocytic myeloid-derived suppressor cell; HLA-DR, human leukocyte antigen DR; M $\Phi$, macrophage; M1, M1 macrophage; M2, M2 macrophage; MDSC, myeloid-derived suppressor cell; M-MDSC, monocytic myeloid-derived suppressor cell; SSC, side scatter. 
Table 1 Differences between G-MDSCs and neutrophils

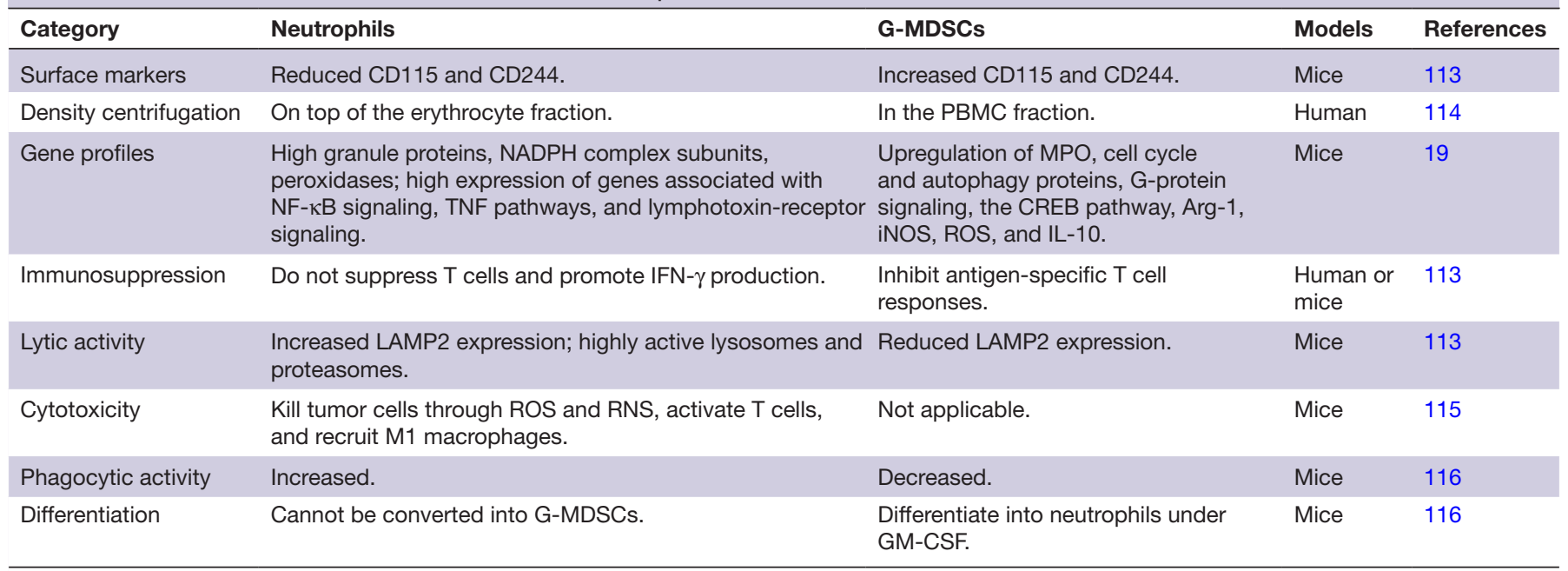

CREB, CAMP-response element binding protein; Arg-1, arginase 1; GM-CSF, granulocyte-macrophage colony stimulating factor; G-MDSCs, granulocytic myeloidderived suppressor cells; IFN- $\gamma$, interferon- $\gamma$; IL-10, interleukin 10; iNOS, inducible nitric oxide synthase; LAMP2, lysosomal-associated membrane protein 2; MPO, myeloperoxidase; NADPH, nicotinamide adenine dinucleotide phosphate; NF-kB, nuclear factor-kappa B; PBMC, peripheral blood mononuclear cell; RNS, reactive nitrogen species; ROS, reactive oxygen species; TNF, tumor necrosis factor.

in $\mathrm{CD}^{+} \mathrm{T}$ cell-mediated colitis mice. ${ }^{4}$ Later, colonic G-MDSCs were described as $\mathrm{Mac}-1^{+} \mathrm{Ly}_{6 \mathrm{C}}{ }^{\text {int }} \mathrm{Gr}-1^{+}$cells. $^{7}$ Colonic M-MDSCs are described as CD $11 b^{+} \mathrm{Ly}_{6 \mathrm{C}} \mathrm{C}^{\mathrm{hi}}$ D $103^{-} \mathrm{CX} 3 \mathrm{CR} 1^{\text {int }}$ or $\mathrm{CD} 11 \mathrm{~b}^{+} \mathrm{CD} 14^{+} \mathrm{CX} 3 \mathrm{CR} 1^{+}$cells that produce high levels of IL-12, IL-23, inducible nitric oxide synthase (iNOS), and tumor necrosis factor- $\alpha$ (TNF- $\alpha){ }^{8}{ }^{9}$ MDSCs in the peripheral blood of patients with CAC have been described as $\mathrm{Lin}^{-1}$ ${ }^{\text {lo }} \mathrm{HLA}_{-D R^{-} \mathrm{CD} 11 \mathrm{~b}^{+} \mathrm{CD}_{3}{ }^{+} \text {cells. }}{ }^{10}$ Human colorectal tumor-infiltrating MDSCs express high levels of CD13 and CD39, and low levels of CD115, CD117, CD124 and programmed death ligand-1 (PD-L1). ${ }^{11}$ We previously sorted human colorectal tumor-infiltrating CD $45^{+} \mathrm{HLA}_{-} \mathrm{DR}^{-} \mathrm{CD} 11 \mathrm{~b}^{+} \mathrm{CD}_{3}{ }^{+}$MDSCs with immunosuppressive functions by flow cytometry. ${ }^{12}$ Figure $1 \mathrm{~A}$ illustrates the current understanding of the surface markers on myeloid cells in patients with CRC and the gating strategy for the identification of colorectalinfiltrating myeloid cells. ${ }^{12-15}$ These markers provide an initial framework and can be complemented by other markers. Human M-MDSCs expressed IL-4R $\alpha .{ }^{16}$ Human G-MDSCs express CD62L, CD54, CD63, PD-L1, and lectin-like oxidized low-density lipoprotein receptor-1 (Lox-1). ${ }^{17}$ In colitis mice and CT-26 tumor-bearing mice, CD49d $\mathrm{d}^{+}$MDSCs are phenotypically and morphologically similar to M-MDSCs and can suppress antigenspecific $\mathrm{T}$ cell responses. CD49d $\mathrm{d}^{-}$MDSCs are similar to G-MDSCs. ${ }^{18}$ These results suggest that CD49d is a promising marker to distinguish G-MDSCs and M-MDSCs. Therefore, human MDSC subsets should be identified based on multiple phenotypic features.

\section{Identification of G-MDSCs versus neutrophils}

G-MDSCs are pathologically activated and relatively immature neutrophils that have been implicated in the pathological regulation of CAC. The transition from G-MDSCs to neutrophils is halted in CAC, which results in the continuous accumulation and activation of G-MDSCs. It is difficult to distinguish G-MDSCs from neutrophils only by phenotypic differences, although the classification of G-MDSCs as CD $11 b^{+} \mathrm{Ly}_{6 \mathrm{G}^{+}} \mathrm{Ly}_{6 \mathrm{C}^{\mathrm{lo}}}$ cells in mice and HLA-DR ${ }^{-}$CD $33^{+}$CD $11 b^{+}$CD $15^{+}$cells in humans has been extensively reported. New knowledge of both G-MDSCs and neutrophils provides an opportunity to distinguish these cell types (table 1 ).

Compared with neutrophils, G-MDSCs exhibit reduced expression of CD16 and CD62L, and increased expression of CD11b, CD66b, CD115, and CD244, and have increased reactive oxygen species (ROS) and arginase 1 (Arg-1) activity. ${ }^{19}$ Neutrophils have higher levels of lysosomal and proteasomal enzymes and stronger cytotoxicity, lytic activity and phagocytosis than G-MDSCs. ${ }^{20}$ Furthermore, neutrophils are on top of the erythrocyte fraction, and G-MDSCs are in the peripheral blood mononuclear cell fraction after density centrifugation. ${ }^{6}$ Finally, the nuclear morphology of neutrophils is hypersegmented, with more than four nuclear lobes (human) or a cloverleaf shape (mouse). G-MDSCs have a horseshoe-shaped or banded-shaped nucleus (human) or a ring-shaped nucleus (mice) (figure $1 \mathrm{C}) .^{20}$

\section{ROLE OF MDSCS/ILCS/THE GUT MICROBIOTA IN THE PATHOGENESIS OF IBD}

CAC was once thought to be a serious complication of IBD. IBD-related chronic non-resolving inflammation is a major driver of CAC. Acute intestinal inflammation is characterized by leukocyte influx followed by macrophage phagocytosis to clear injurious stimuli, leading to 


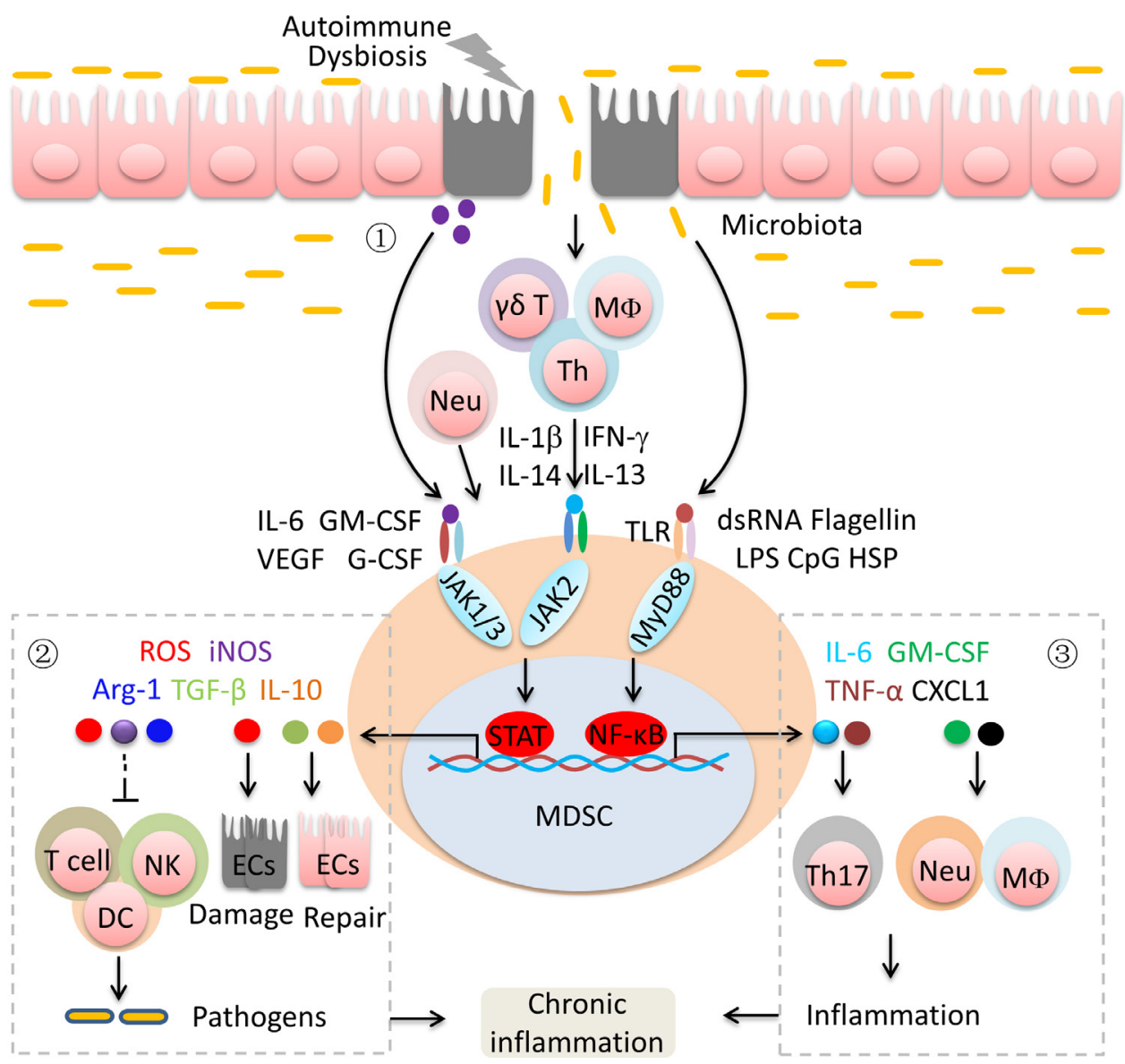

Figure 2 MDSCs-mediated chronic inflammation in inflammatory bowel disease. (1) Immunological stress, IEC damage and the microbiome lead to the recruitment and activation of MDSCs. (2) MDSCs alternately induce IEC damage and repair by secreting effector molecules. MDSC-mediated immunosuppression results in the inefficient removal of micro-organisms. (3) MDSCs promote the differentiation and recruitment of inflammatory cells. Arg-1, arginase 1; CXCL1, C-X-C motif chemokine ligand 1; CpG, cytosine phosphate-guanosine; DC, dendritic cell; ECs, epithelial cells; G-CSF, granulocyte colony-stimulating factor; GM-CSF, granulocyte-macrophage colony stimulating factor; HSP, heat shock protein; IEC, intestinal epithelial cell; IFN- $\gamma$, interferon- $\gamma$; IL, interleukin; iNOS, inducible nitric oxide synthase; LPS, lipopolysaccharide; MDSCs, myeloid-derived suppressor cells; Neu, neutrophils; NK, natural killer cell; ROS, reactive oxygen species; TGF- $\beta$, transforming growth factor- $\beta$; Th, T helper T cell; TLR, Toll-like receptor; TNF- $\alpha$, tumor necrosis factor- $\alpha$; VEGF, vascular endothelial growth factor.

resolution and tissue homeostasis. In patients with IBD, although innate immune-mediated responses to bacteria resolve within days, these stimuli activate the adaptive immune response and trigger a second wave of leukocyte influx into tissues. ${ }^{21}$ MDSCs are an important component of these cells and play a pivotal role in the pathogenesis of IBD by secreting proinflammatory cytokines, as well as interacting with the host immune system and the gut microbiota.

\section{Role of MDSCs in the pathogenesis of IBD}

MDSCs were once regarded as endogenous antagonists of immune system functionality in mucosal inflammation due to their immunosuppressive effects on effector $\mathrm{T}$ cells. MDSCs are recruited and activated in intestinal tissue when challenged with inflammation. However, colonic MDSCs fail to inhibit the inflammatory response and instead promote effector $\mathrm{T}$ cell expansion. ${ }^{7-9}$ IEC-derived and activated immune cell-derived cytokines and gut microbiota-derived factors lead to the recruitment and activation of MDSCs in the intestine (figure 2). ${ }^{22}{ }^{23}$ In chronic colitis mice, colonic G-MDSCs acquire stimulatory antigen-presenting functions and induce $\mathrm{T}$ cell activation and IL-17 production. ${ }^{7}$ Adoptively transferred $\mathrm{CD}_{11 \mathrm{~b}}{ }^{+}$Ly6 $\mathrm{C}^{\mathrm{hi}}$ cells are converted into proinflammatory cells and promote intestinal inflammation. ${ }^{89}$ Human HLA-DR ${ }^{-/ 10} \mathrm{CD} 33^{+} \mathrm{C}-$ $\mathrm{D} 15^{+} \mathrm{CD} 14^{-}$MDSCs from the peripheral blood of patients with IBD not only fail to suppress the autologous $\mathrm{T}$ cell response but also enhance $\mathrm{T}$ cell proliferation. ${ }^{24}$ Furthermore, activated MDSCs inhibit the antigen uptake and processing by DCs and subsequent $\mathrm{CD}^{+} \mathrm{T}$ cell proliferation and activation, which leads to inadequate clearance of pathogenic bacteria at sites of bacterial penetration, resulting in a sustained inflammatory stimulus (figure 2). ${ }^{25}{ }^{26}$ MDSC-derived ROS are the major inducers of the IEC damage, but 


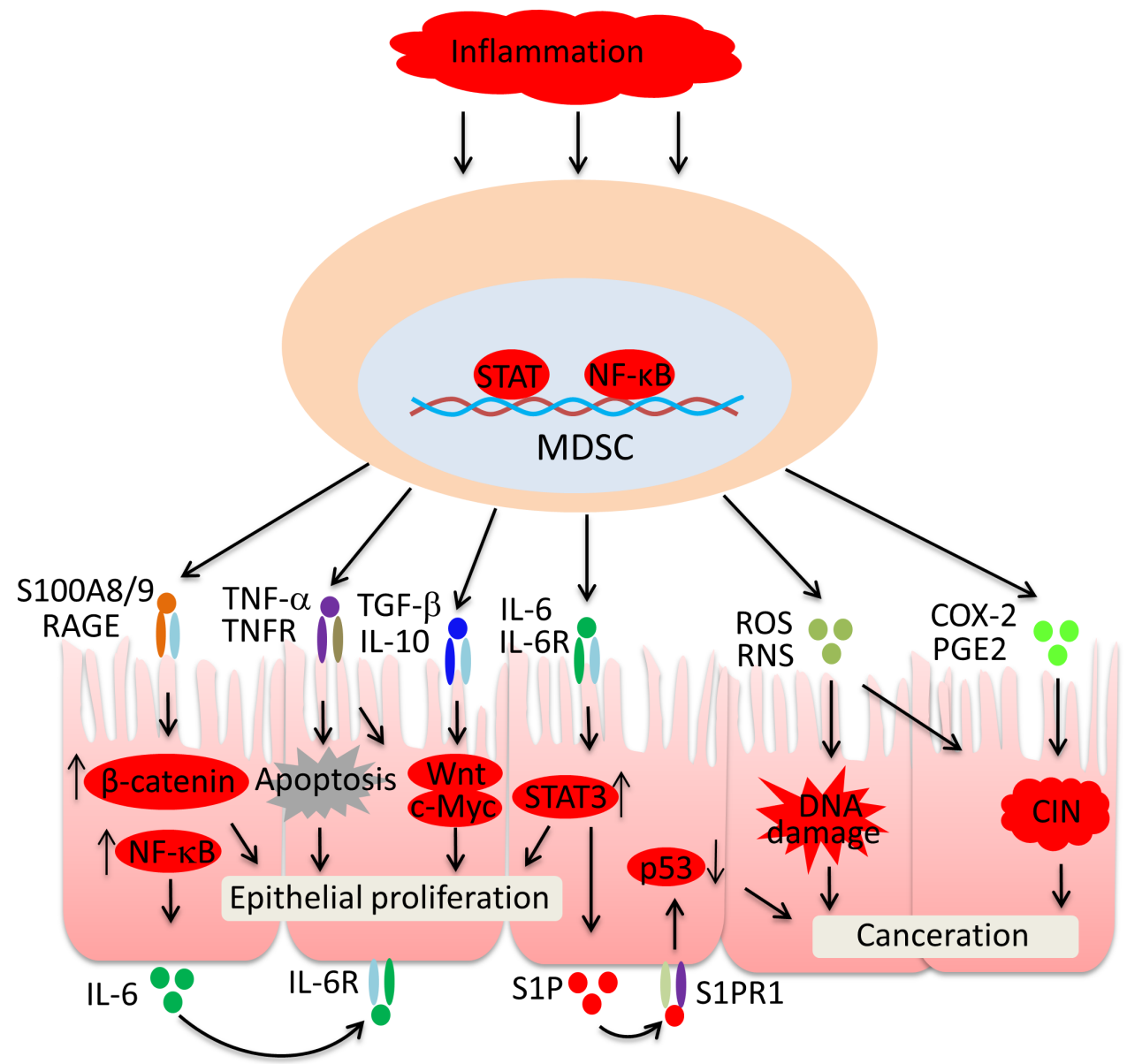

Figure 3 MDSC-mediated events in intestinal epithelial dysplasia. S100A8/9 upregulates IL-6 production via the RAGE/NF$\kappa B$ pathway. IL-6-dependent activation of STAT3 promotes IEC proliferation and S1P production. S1P inhibits p53 expression via S1PR1. TNF- $\alpha$ drives IEC apoptosis, which in turn accelerates the proliferation of IECs. TNF- $\alpha$, S100A8/9, TGF- $\beta$ and IL-10 activate Wnt/ $\beta$-catenin in IECs, which promotes mucosal regeneration. Persistent exposure to ROS and COX-2-derived PGE2 leads to DNA damage and CIN in IECs. CIN, chromosomal instability; COX-2, cyclo-oxygenase-2; IEC, intestinal epithelial cell; IL, interleukin; MDSC, myeloid-derived suppressor cells; NF-кB, nuclear factor-kappa B; PGE2, prostaglandin E2; RAGE, receptor of advanced glycation end products; RNS, reactive nitrogen species; ROS, reactive oxygen species; S1P, sphingosine 1-phosphate; S1PR1, sphingosine 1-phosphate receptor 1; STAT3, signal transducer and activator of transcription 3; TGF- $\beta$, transforming growth factor- $\beta$; TNF- $\alpha$, tumor necrosis factor- $\alpha$; TNFR, tumor necrosis factor receptor.

MDSC-derived transforming growth factor- $\beta$ (TGF- $\beta$ ) promotes IEC repair. ${ }^{27}$ These double effects drive chronic inflammation. Under the colonic inflammatory milieu, MDSCs downregulate the expression of CCAAT/enhancer-binding protein beta $($ CEBP $\beta)$, a critical transcription factor associated with the suppressive function of MDSCs, and secreted increased levels of proinflammatory molecules. ${ }^{24}$ In addition, MDSCderived proinflammatory molecules, such as IL-6, TNF- $\alpha$, granulocyte-macrophage colony stimulating factor (GM-CSF) and C-X-C motif chemokine ligand 1 (CXCL1), promote Th17 cell differentiation and the influx of macrophages and neutrophils, resulting in a strong inflammatory response (figure 2).$^{28}$ Therefore, MDSCs are the key factors in the continual reinitialization of chronic intestinal inflammation over long periods of time and the targetable link between acute inflammation and chronic inflammation in IBD.

\section{Role of ILCs in the pathogenesis of IBD}

Susceptibility loci for IBD are closely linked to ILC function and the depletion of ILCs reduces the severity of colonic inflammation. ${ }^{29}{ }^{30}$ ILC1s and ILC3s are enriched in the intestinal tissue of patients with $\mathrm{CD}$ and promote intestinal inflammation by producing interferon- $\gamma($ IFN- $\gamma)$ and IL-17. ${ }^{30}$ Fontolizumab and secukinumab, two humanized neutralizing monoclonal antibodies (mAbs) against IFN- $\gamma$ and IL-17, respectively, can effectively inhibit CD activity. ${ }^{31}{ }^{32}$ ILCs isolated from patients with active CD showed increased gene expression of IL-23R, which is a key ILC3 cytokine receptor. ${ }^{33}$ IL-23 regulates the selective accumulation of the ILC3 population, which is characterized by inflammatory cytokine expression and is associated with intestinal inflammation in CD. ${ }^{33}$ IL-23 produced by MDSCs is a driver of inflammation and tumors. ${ }^{34}$ Thus, IL-23 from MDSCs may promote the expansion of ILC3s through IL-23R and is expected to be a target for the treatment of IBD and CAC. 


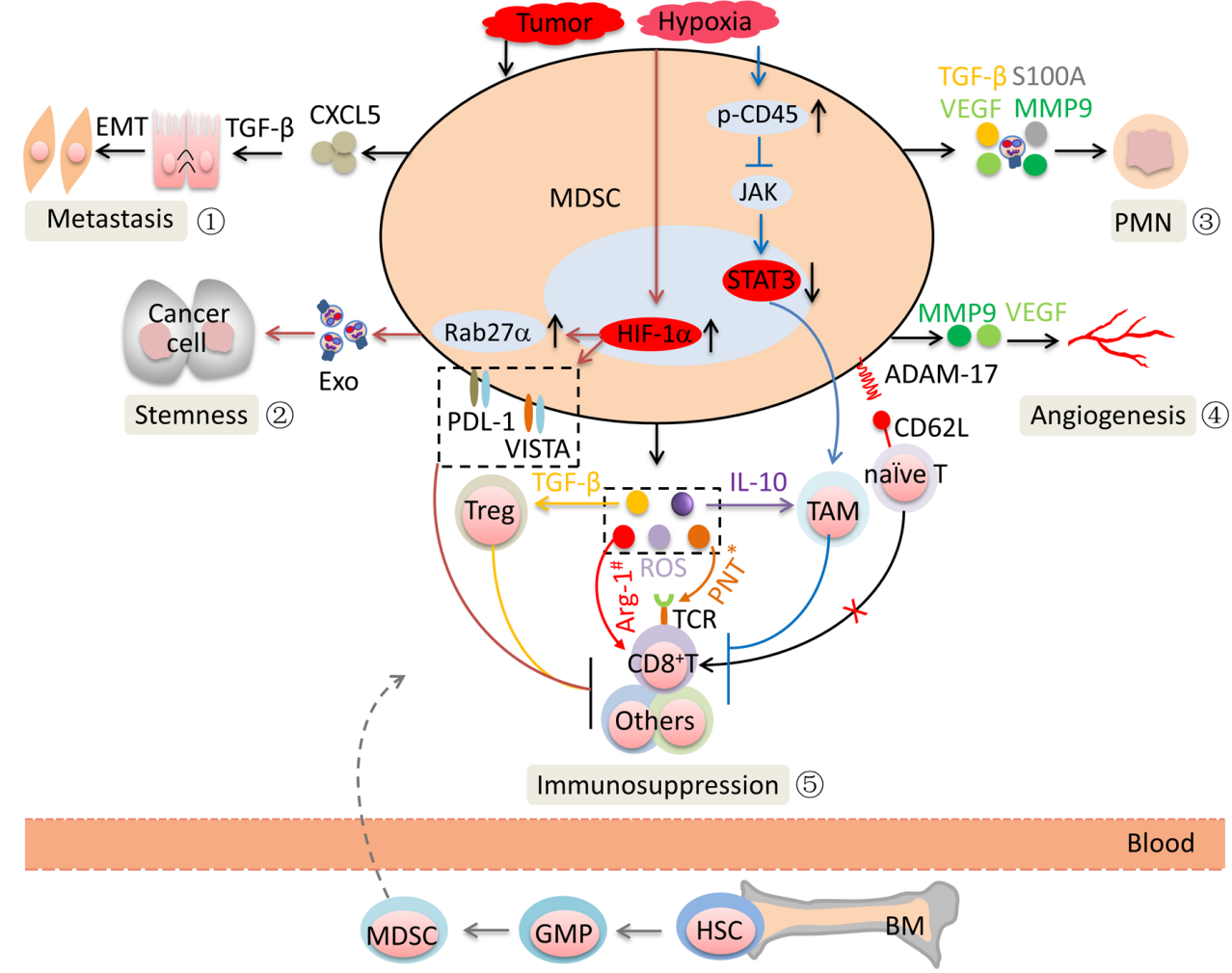

Figure 4 MDSC activity in the promotion of CAC. (1) MDSCs support EMT via TGF- $\beta$ signaling pathways. (2) MDSCs promote the stemness of CAC cells via exosomes secretion. (3) MDSCs promote the formation and evolution of the PMN. (4) MDSCs promote blood vessel formation. (5) MDSCs suppress antitumor immune responses. 'Represents Ag-specific suppression; \#represents non-specific suppression. ADAM-17, A disintegrin and metalloproteinase domain 17; Ag, antigen; Arg-1, arginase 1; BM, bone marrow; CAC, colitis-associated cancer; CXCL1, C-X-C motif chemokine ligand 1; EMT, epithelial-mesenchymal transition; Exo, exosome; GMP, granulocyte-monocyte precursors; HIF-1 $\alpha$, hypoxia-inducible factor $1 \alpha$; HSC, hematopoietic stem cell; IL, interleukin; JAK, Janus kinase; MDSCs, myeloid-derived suppressor cells; MMP9, matrix metallopeptidase 9; PD-L1, programmed death ligand-1; PMN, premetastatic niche; PNT, peroxynitrite; ROS, reactive oxygen species; STAT3,signal transducerand activator of transcription 3; TAM, tumor-associated macrophages; TCR, T cell receptor; TGF- $\beta$, transforming growth factor- $\beta$; Treg, regulatory T cell; VEGF, vascular endothelial growth factor; VISTA, V-domain Ig suppressor of T cell activation.

Intestinal ILC2s induced strong type 2 innate inflammation by secreting cytokines, such as IL-4, IL-5, and IL-13. ${ }^{35}$ Previous studies showed that ILC2-derived IL-4, IL-5, and IL-13 were linked to impaired epithelial barrier function in the gut and drove UC. ${ }^{36}{ }^{37}$ G-MDSCs effectively suppressed the cytokine production of ILC2s in allergy-induced airway inflammation models, thereby alleviating airway inflammation, ${ }^{38}$ although the potential relationship between ILC2s and MDSCs in IBD remains largely unknown. Interestingly, ILC2s could prevent acute gastrointestinal graft-versus-host disease following hematopoietic stem cell transplantation, which was associated with ILC2-derived IL-13 promotion of MDSCs and reductions in proinflammatory Th1 and Th17 cells. ${ }^{39}$ In the context of acute promyelocytic leukemia, activated ILC2s promoted M-MDSC activation through IL-13 secretion and were associated with poor tumor control. ${ }^{40}$ Thus, ILC2s may limit antitumor immune responses indirectly by modulating MDSC functions. However, ILC-derived IL-22 and amphiregulin play protective roles in IBD by promoting mucus secretion and epithelial cell repair. ${ }^{42}$ Thus, the crosstalk between MDSCs and ILCs plays an important and complex role in the pathogenesis of IBD. There are more regulatory mechanisms of MDSCs in the pathogenesis of CAC than those of ILCs, although additional regulatory effects of ILCs in cancer are being discovered. Investigating the relationship between ILCs and MDSCs will help clarify the regulatory mechanism of ILCs and MDSCs in IBD, which could provide therapeutic benefit in the treatment of IBD and CAC.

\section{Role of the microbiota in the pathogenesis of IBD}

There are already abundant data confirming the importance of the gut microbiota in the pathogenesis of IBD and CAC by inducing host immune response disturbance and chronic inflammation. Some pathogenic microbiota, such as Mycobacterium avium subspecies paratuberculosis, ${ }^{43}$ Cytomegalovirus, ${ }^{44}$ and adherent-invasive Escherichia coli ${ }^{45}$ participate directly in the pathogenesis of IBD. Furthermore, reduced microbial diversity is an important reason for IBD. The loss of Bacteroides suppresses the conversion of non-digestible dietary fiber into short-chain fatty acids. ${ }^{46}$ A reduction in Clostridium enhances mucosal permeability, which results in the exposure of antigens and bacterial Toll-like receptor ligands, thereby activating pathogenic immune 
Table 2 Possible therapy strategies for CAC based on MDSC regulation

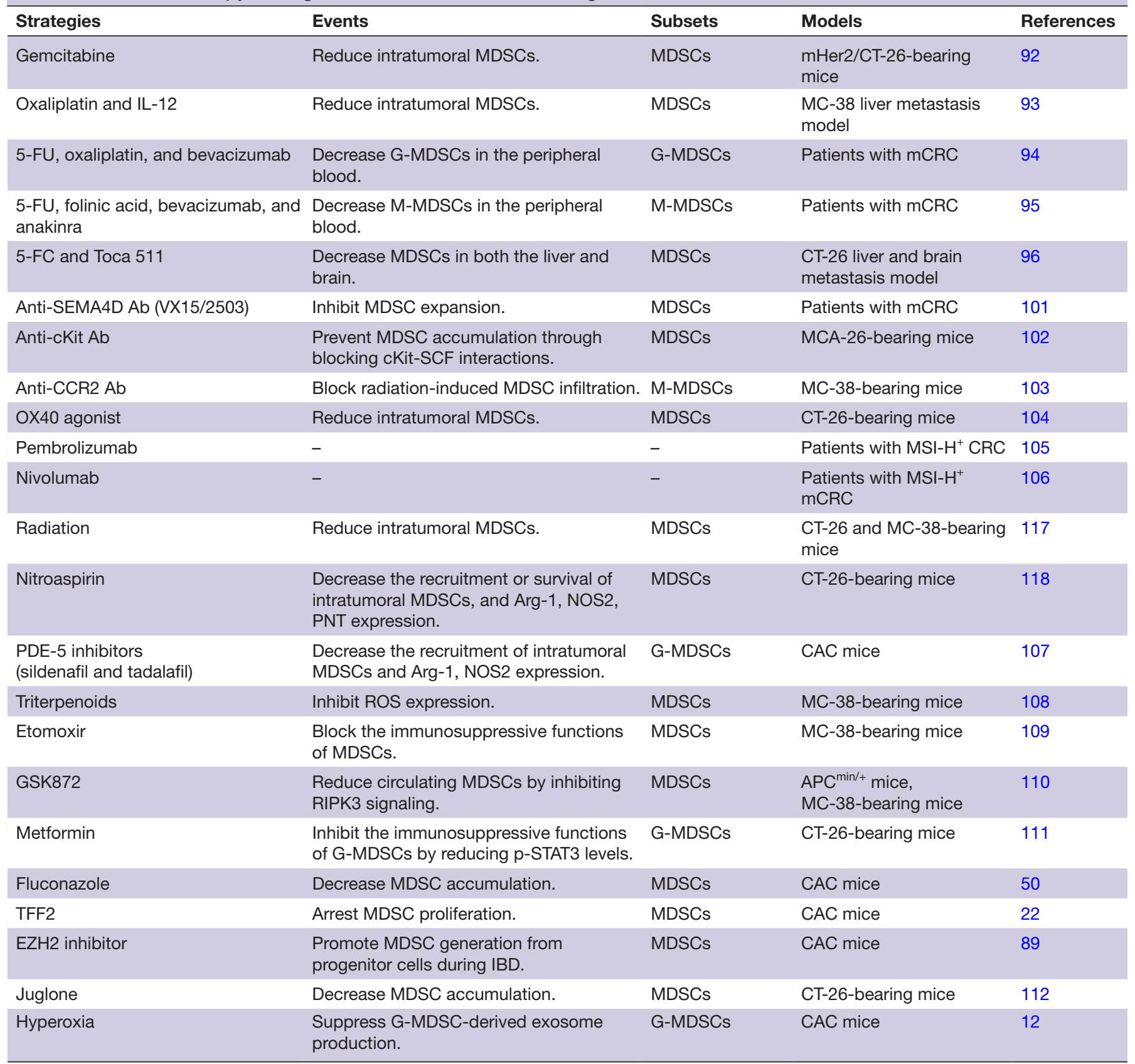

$\mathrm{Ab}$, antibody; Arg-1, arginase 1; CAC, colitis-associated cancer; CCR2, chemokine (C-C motif) receptor 2; c-Kit, cellular kit proto-oncogene; EZH2, enhancer of zeste homolog 2; 5-FC, 5-fluorocytosine; 5-FU, 5-fluorouracil; G-MDSCs, granulocytic MDSCs; IBD, inflammatory bowel disease; IL, interleukin; mCRC, metastatic colorectal cancer; MDSCs, myeloid-derived suppressor cells; M-MDSCs, monocytic MDSCs; MSI-H, microsatellite instability-high; OX40, oxford 40; PDE-5, phosphodiesterase 5; PNT, peroxynitrite; RIPK3, receptor interacting protein kinase 3; ROS, reactive oxygen species; SEMA4D, semaphorin 4D; STAT3, signal transducer and activator of transcription 3; TFF2, trefoil factor 2.

responses. ${ }^{47}$ Fecal microbiota transplantation can increase fecal microbial diversity in patients with active UC and is a promising treatment to induce remission. ${ }^{48}$ Interestingly, IBD-associated microbiota can induce the accumulation and proinflammatory functions of MDSCs. The combined action of enterotoxigenic Bacteroides fragilis and IL-17 on IECs promoted the differentiation of proinflammatory M-MDSCs. ${ }^{49}$ This may explain why bone marrow-MDSCs potently suppress $\mathrm{CD} 4^{+} \mathrm{T}$ cell responses but fail to control colitis-associated immune responses in vivo. Bone marrow cells incubated with Candida tropicalis exhibited MDSCs features. ${ }^{50}$ Mice deficient in fungal killing exhibit dysbiosis and have increased susceptibility to colitis and CAC. ${ }^{50}$ The role of the gut microbiota in shaping MDSC phenotype and function remains to be further studied. The important task is to identify the unique microbiota that drives IBD and the relationship of the gut microbiota with myeloid cells, which could help in the development of personalized intervention 
strategies that correct abnormalities and induce sustainable treatment responses in patients with IBD.

\section{EFFECT OF MDSCS ON IECS DURING THE DEVELOPMENT OF CAC}

Dysplasia has been linked to CAC initiation, in which disease promotion is driven by the sustained proliferation of IECs and genomic alterations in IECs. MDSCs are already the targetable link between IBD and CAC. Here, we review the current knowledge concerning how MDSCs participate in the CAC initiation by mediating proliferation signals and DNA damage in IECs (figure 3).

Under normal conditions, signal transducer and activator of transcription 3 (STAT3) plays an important role in maintaining intestinal epithelial homeostasis by promoting IEC proliferation. Under IBD conditions, MDSC-derived IL-6 promotes the continuous activation of STAT3 through the IL-6R signaling pathway, which results in uncontrollable IEC proliferation. ${ }^{51}$ Additionally, IL-6-activated STAT3 promotes the secretion of sphingosine 1-phosphate (S1P), which binds S1P receptor 1 and plays a critical role in IEC dysplasia by inhibiting p53 expression. ${ }^{52}$ Receptor of advanced glycation end products (RAGE) initiates cellular activation of multiple pathways and is multifunctional in the carcinogenesis of various solid tumors. ${ }^{53}$ Extracellular S100A8/9 derived from MDSCs activates $\beta$-catenin via RAGE, which initiates dysplasia in IECs. ${ }^{54}$ Interestingly, the activation of the RAGE signaling pathway also promotes IL-6 production, which contributes to STAT3 activation and p53 expression inhibition. ${ }^{55}$ Therefore, MDSC-derived S100A8/9 plays an important role in dysplasia and CAC initiation. TNF- $\alpha$ is a major cytokine that mediates MDSC functions in IBD. The loss of tumor necrosis factor receptor (TNFR) in IECs blunts Wnt signaling and wound healing in IBD mice, indicating an integral role of TNF- $\alpha$ in mucosal regeneration. ${ }^{56}$ TNF- $\alpha$ activates $\beta$-catenin via protein kinase $\mathrm{B}$ and induces Wnt expression in crypt base stem cells. ${ }^{57}$ Additionally, TNF- $\alpha$ drives IEC apoptosis during colitis, which in turn accelerates the proliferation of IECs. ${ }^{58}$ IL-10 and TGF- $\beta$, the key molecules that mediate MDSC immunosuppression during colitis, promote IEC proliferation by activating Wnt signaling and promoting c-Myc expression in IECs. ${ }^{59}$ Chronic exposure to ROS promotes cell canceration by promoting DNA damage and chromosomal instability (CIN) in IECs. Increased oxidative stress leads to p53 mutations, which initiate dysplasia in IECs. Genetic and epigenetic changes in DNA further contribute to carcinoma at the later stage of atypical hyperplasia. During IBD, excessive MDSC-derived ROS may promote the transition from chronic inflammation to dysplasia by damaging IEC DNA. ${ }^{60}$ The levels of cyclo-oxygenase-2 (COX-2) and COX-2-derived prostaglandin E2 (PGE2) are significantly elevated in patients with IBD. ${ }^{61}$ MDSCs in preneoplastic colon adenomas commonly express COX-2 and PGE2. PGE2 initiates CIN in IECs, enhances the stemness of tumor cells, transforms
IECs into cancer-initiating cells, and drives CAC. ${ }^{62}$ Thus, MDSCs may promote CAC through COX-2 and PGE2.

MDSC-related oxidative stress, aberrant inflammation, and tissue repair signaling lead to the activation of p53 mutations and Wnt/ $\beta$-catenin, which initiate dysplasia in IECs. MDSCs also contribute to CAC at the later stage of IBD by promoting genetic and epigenetic changes in DNA.

\section{ROLE OF MDSCS IN CAC PROGRESSION}

It is well known that MDSCs promote CAC through immune suppression. However, the rescue of antitumor immunity is insufficient to achieve the expected clinical effect, although the results of animal experiments showed that targeting MDSCs is an emerging opportunity for enhancing the effectiveness of anti-CAC therapy. Researchers have found that MDSCs also promote CAC progression through a non-immunosuppressive effect.

\section{Immunosuppression}

Under CAC conditions, stromal cell-derived and tumorderived factors mobilize myeloid progenitors to develop the phenotypic features of MDSCs and acquire suppressive activity toward immune cells through multiple mechanisms. CD8 ${ }^{+}$cytotoxic T lymphocytes (CTLs) are central players in controlling cancer. ${ }^{63}$ MDSCs predominantly suppress CTLs via effector molecules such as Arg-1, ROS, and nitric oxide (NO). Peroxynitrite formed by the cooperative activity of ROS and NO leads to the nitration of tyrosines in the $\mathrm{T}$ cell receptor (TCR)-CD8 complex, which disrupts the conformational flexibility of the TCRCD8 complex. The damaged TCR-CD8 complex cannot interact with peptide-loaded major histocompatibility complex I (MHC-I), which results in the unresponsiveness of $\mathrm{CD}^{+} \mathrm{T}$ cells to antigen-specific stimulation. ${ }^{6465}$ MDSCs directly cleave CD62L on the surface of naive $\mathrm{T}$ cells by expressing A disintegrin and metalloproteinase domain 17 (ADAM17), which suppresses naive T cell differentiation into CTLs (figure 4) ${ }^{66}$ MDSCs also induce regulatory $\mathrm{T}$ cell (Treg) expansion by producing TGF- $\beta .^{67}$ Tumorinfiltrating MDSCs are exposed to the hypoxic microenvironment, which results in an increase in hypoxia-inducible factor $1 \alpha$ (HIF-1 $\alpha) .{ }^{12}$ HIF-1 $\alpha$ promoted the production of Arg-1 and iNOS and the upregulation of inhibitory V-domain Ig suppressor of T cell activation and PD-L1, which promoted MDSC-mediated T cell suppression. ${ }^{68} 69$ The hypoxic microenvironment causes the activation of CD45 protein tyrosine phosphatases, which results in the downregulation of STAT3 activity and promotes M-MDSC differentiation to tumor-associated macrophages. ${ }^{70}$ These alterations result in the potent non-specific immunosuppressive activity of MDSCs within the tumor.

\section{Non-immunosuppression}

MDSCs have been shown to play pivotal and intricate roles in promoting CAC metastasis. Great efforts focusing on the regulatory network of MDSCs in CAC have been made. We will discuss some new advances in the field, 
documenting an increase in the sphere of influence of MDSCs (figure 4).

Epithelial-mesenchymal transition (EMT) plays pivotal roles in CAC metastasis. The TGF- $\beta /$ Smad signaling pathway is an essential driver of EMT. In a model of uveal melanoma, G-MDSCs are recruited to primary tumors by CXCL5 and then induce EMT by the TGF- $\beta$, epidermal growth factor, and hepatocyte growth factor signaling pathways, which promote cancer cell dissemination. ${ }^{71}$ MDSC-derived matrix metallopeptidase 9 (MMP9) increases the bioavailability of vascular endothelial growth factor and promotes angiogenesis, which was crucial in CAC metastasis. The promotion of tumor metastasis by forming a premetastatic niche (PMN) is widely accepted. In CRC liver metastasis mice, MDSCs promote liver-specific metastasis by promoting PMN formation. ${ }^{10}$ In other tumor models, MDSC-derived prometastatic proteins such as Bv8, MMP9, S100A8/9, TGF- $\beta$, and inhibitory molecules participate in multiple stages of PMN formation and evolution by increasing vascular permeability and the degradation of tight junction proteins, suppressing the immune response, and promoting the formation of an inflammatory microenvironment. ${ }^{72}$ Our previous research showed that G-MDSCs promoted CAC cell stemness through exosomal S100A9. ${ }^{12}$

\section{TARGETING MDSCS FOR CAC THERAPY}

The multifunctional features of MDSCs in IBD/CAC force us to comprehensively assess the role of MDSCs in the prevention and treatment of CAC. Here, we summarize the microenvironmental characteristics of $\mathrm{CAC}$, the role of MDSCs in the response to anti-inflammatory IBD treatment, and the possible approaches targeting MDSCs for CAC therapy.

\section{Microenvironmental characteristics of CAC}

CAC and sporadic colorectal cancer (sCRC) are the two major forms of CRC. Chronic inflammation and immune dysregulation predispose IECs to dysplasia and eventually lead to the development of CAC in patients with IBD, whereas the accumulation of mutations in oncogenes and tumor suppressor genes drives the initiation of sCRC. The unique initiation mechanisms lead to the unique and complex microenvironment of CAC. First, sustained chronic inflammation leads to DNA damage that exceeds the capacity of DNA repair, which is the major mechanism responsible for the microsatellite instability (MSI) phenotype ${ }^{73}$ MSI is more likely to occur in patients with CAC than in patients with sCRC (approximately 50\% compared with $15 \%){ }^{73-77}$ The microenvironment of $\mathrm{MSI}^{+}$CRC contains strong Th1 and CTL components. The increased number of tumor 'neoantigens' created by the chronic inflammation-driven high mutational load may be an important factor. ${ }^{78}$ Multiple immune checkpoints, such as cytotoxic T-lymphocyte associated protein 4 (CTLA-4), programmed cell death protein 1 (PD-1), and PD-L1, are highly elevated in $\mathrm{MSI}^{+} \mathrm{CRC}$ relative to microsatellite stability (MSS) CRC. ${ }^{79}$ Additionally, chronic inflammation damages epithelial integrity and increases intestinal permeability in patients with CAC, which allows luminal microbiota to freely enter the lamina propria (LP) and promote tumor progression. ${ }^{80}$

\section{Role of MDSCs in the response to anti-inflammatory IBD treatment}

Persistent chronic inflammation plays an important role in the initiation of CAC. Anti-inflammatory treatment is an important means to prevent the development of CAC. In experimental mouse models, a variety of antiinflammatory agents, such as zileuton, ${ }^{81}$ infliximab, ${ }^{82}$ and omeprazole, ${ }^{83}$ have been suggested to prevent the occurrence of CAC. Earlier and intensive anti-inflammatory therapy should be considered for either mitigating clinical courses or preventing the development of CAC in high-risk patients with IBD.

Relapse and intolerance to existing drugs, such as antibiotics, corticosteroids, and aminosalicylate, always occur during the treatment of IBD. Enormous efforts have been made to develop new treatment strategies based on immunosuppressive interventions. MDSCs are one cell type with a well-recognized role in limiting immune reactions and play an important role in the response to IBD treatment. Dexamethasone suppresses HIF-1 $\alpha$-dependent glycolysis in MDSCs through glucocorticoid receptor signaling and thus promotes the immunosuppressive activity of MDSCs. ${ }^{84}$ INK128, an mTOR kinase inhibitor, is in clinical development. In dextran sodium sulfate (DSS)-induced murine colitis, INK128 can maintain the immature state of MDSCs by elevating S100A8/9 expression and reducing the production of inflammatory cytokines. ${ }^{85}$ Glatiramer acetate enhances IL-10 and TGF- $\beta$ secretion while reducing IL-23 and IL-6 secretion from MDSCs via the recognition of paired Ig-like receptor B, thus favoring conditions that suppress Th17 maturation but enhance Treg induction. ${ }^{86}$ Atorvastatin promotes the expansion of G-MDSCs, which suppress $\mathrm{T}$ cell responses via NO production. Transfer of these G-MDSCs attenuates chronic colitis. ${ }^{87}$ In an IBD model induced by the bacterium Helicobacter hepaticus, oral administration of diallyl trisulfide reduces colon inflammation by limiting the recruitment of G-MDSCs in the colon. ${ }^{88}$ GSK343, an inhibitor of enhancer of zeste homolog 2 (EZH2), shows beneficial effects on DSS-induced colitis by increasing the number of MDSCs in the LP. ${ }^{89}$ Therefore, the immunosuppressive effect of MDSCs plays an important role in limiting IBD-related inflammation and may be used as an indicator to assess the response to IBD therapeutic strategies.

Indeed, in addition to chronic inflammation, the initiation of CAC is also related to cumulative genetic changes, abnormal immune regulation, and intestinal microfloral imbalance. Anti-inflammatory preparations-mediated MDSC expansion is also likely to shape CAC. MDSCderived ROS participate in CAC initiation by mediating proliferation signals and DNA damage in IECs, which 
promotes the transition from inflammation to dysplasia. ${ }^{60}$ MDSC-derived S100A8/9 activated $\beta$-catenin via RAGE, which initiates dysplasia in IECs. ${ }^{54}$ Furthermore, MDSCs suppress the body's immune response, which is beneficial for abnormally hyperplastic IECs to escape immune surveillance. MDSC-mediated chronic inflammation and an immunosuppressive microenvironment are suitable for the settlement and survival of tumor cells. ${ }^{7}$ Persistent application of antibiotics and glucocorticoid drugs leads to imbalance in the gut microbiota, which is a key event leading to chronic tissue injury and CAC initiation. Therefore, anti-inflammatory treatment is expected to become a specific preventive measure for CAC. However, developing true anti-inflammatory treatments to prevent CAC remains inherently challenging due to anti-inflammatoryrelated side effects and potential risks.

\section{Role of chemotherapy drugs in MDSC depletion}

The majority of chemotherapy drugs have been shown to reduce the tumor infiltration of MDSC (table 2). Sunitinib potently decreased MDSC accumulation and rescued T cell inhibition in colon cancer cell-bearing mice ${ }^{90}$ MDSCs are the major components of the tumor microenvironment in both murine models of patients with colon cancer and CRC, although the microenvironments are different. Sunitinib-mediated MDSC elimination is beneficial in restoring antitumor immunity in patients with CAC. However, sunitinib malate combined with 5-fluorouracil/ leucovorin/irinotecan (FOLFIRI) chemotherapy did not significantly improve progression-free survival in patients with metastatic colorectal cancer (mCRC) compared with that of FOLFIRI alone. ${ }^{91}$ The sensitivity of CRC cells themselves may be the main reason for the failure of sunitinib in clinical trials. Additionally, in patients with CAC, the microenvironment in which MDSCs exist is more complex due to the microbiota, dietary components and metabolites. In colon cancer cell-bearing mice, both gemcitabine and oxaliplatin induced protective antitumor immunity by eliminating MDSCs. ${ }^{92}{ }^{93}$ 5-Fluorouracil (5-FU) has a good effect on MDSC depletion. Bevacizumab in combination with 5-fluorouracil/leucovorin calcium/oxaliplatin (FOLFOX) or leucovorin calcium/5-fluorouracil (LV5FU2) and anakinra could decrease the number of G-MDSCs or M-MDSCs in patients with mCRC. ${ }^{94} 95$ In CT-26 metastatic mice, 5-fluorocytosine in combination with Toca 511 significantly decreased the number of MDSC in both the liver and brain in mCRC mice. ${ }^{96} \mathrm{~A}$ phase I study of Toca 511 and Toca FC in solid tumors, including mCRC, has been completed and showed a promising novel treatment strategy. ${ }^{97}$ Gemcitabine and 5-FU activate the pyrin domain containing-3 protein (NLRP3)-dependent caspase-1 activation complex in MDSCs, resulting in the production of IL-1 $\beta$, which limits the antitumor efficacy. Gemcitabine and 5-FU exert an improved antitumor effect when combined with an IL-1 receptor antagonist. ${ }^{98}$ In murine mammary cancer models, anthracyclines not only eliminated peripheral and intratumoral MDSCs but also caused a shift toward a more differentiated phenotype featuring upregulated Gr-1 and downregulated CD11b in MDSCs. ${ }^{99} 100$ Thus, anthracycline may have synergistic effects with other anti-CAC agents by effectively depleting MDSCs, which provides a promising strategy for potential CAC therapy.

\section{Role of antibodies in MDSC inhibition}

Antibodies have been found to block the expansion of MDSCs in CRC (table 2). Preclinical studies demonstrated that blocking semaphorin 4D (SEMA4D) inhibited MDSC expansion in patients with CRC. ${ }^{101}$ Phase I clinical studies of anti-SEMA4D mAb (VX15/2503) in mCRC are underway (NCT03373188). In MCA-26-bearing mice, blocking the stem cell factor (SCF) receptor-SCF interaction by anticKit (anti-cellular kit proto-oncogene) mAbs significantly reduced MDSC expansion and restored the proliferative responses of tumor-infiltrating $\mathrm{T}$ cells. ${ }^{102}$ An anti-chemokine (C-C motif) receptor 2 (CCR2) antibody blocked radiationinduced M-MDSC infiltration. ${ }^{103}$ In CT-26-bearing mice, the oxford 40 (OX40) agonist antibody significantly decreased the expression of TGF- $\beta$ in MDSCs. ${ }^{104}$

MSI is a main type of genomic instability factor. Carcinogenesis and malignant potential in CAC are closely associated with MSI caused by TGFßRII mutations and hMLH1 hypermethylation. Chronic inflammation and folate deficiency lead to high frequencies of MSI in patients with IBD and CAC. Brentnall et $a l^{73}$ detected MSI in $46 \%$ of high-grade dysplasias, in $40 \%$ of carcinomas, and in $50 \%$ of non-neoplastic mucosa. Ishitsuka et $a l^{76}$ found MSI in $8 \%$ of dysplasias, in $50 \%$ of carcinomas, and in $9 \%$ of non-neoplastic mucosa from patients with UC who had long durations of neoplasms. Schulmann $e t$ $a l^{77}$ detected MSI in $67 \%$ of high-grade dysplasias and in $67 \%$ of carcinomas. Therefore, approximately half of the CAC cases are MSI-H. MSI- $\mathrm{H}^{+}$is detected in only approximately $15 \%$ of sCRC cases. $^{73}{ }^{75}$ MSI-H tumors selectively upregulate the expression of various immune checkpoint factors, such as PD-1, PD-L1, and CTLA-4. ${ }^{79}$ Humanized anti-PD-1/ PD-L1 mAbs have shown promising results in patients with CRC, and the clinical efficacy is especially significant in $\mathrm{MSI}^{+}$ CRC. ${ }^{105} 106$ Therefore, CAC is more likely to respond effectively to immunotherapy than sCRC, although this has not been analyzed separately. PD-1/PD-L1 mAbs are another available drug for CAC immunotherapy, although the exact clinical efficacy needs to be further defined. It is worth noting that non-neoplastic mucosa and high grade-dysplasia in CAC have also been found to have MSI. Early application of antiPD-1/PD-L1 mAbs may be beneficial for either mitigating clinical courses or preventing the ultimate development of CAC in high-risk patients with IBD.

\section{Role of small molecule inhibitors in MDSC inhibition}

The alternative strategy to suppress MDSCs is to inhibit their effector molecules and catabolic enzymes (table 2). In CAC mice, Arg-1 and iNOS can be blocked by nitroaspirin and phosphodiesterase-5 inhibitors. ${ }^{107}$ In MC-38-bearing mice, both triterpenoids and etomoxir dampened the immunosuppressive effects of MDSCs by reducing ROS secretion. ${ }^{108} 109$ In $\mathrm{APC}^{\mathrm{min} /+}$ mice and MC-38-bearing mice, GSK872 inhibited 
MDSC to produce IL-23, IL-1 $\beta$, and COX-2, which inhibited tumor growth. ${ }^{110}$ Metformin downregulated the inhibitory functions of G-MDSCs by reducing STAT3 phosphorylation levels in MC-38-bearing mice. ${ }^{111}$ In addition, fluconazole, trefoil factor 2, and an EZH2 inhibitor have been found to suppress CAC susceptibility by decreasing MDSC accumulation. $^{22} 5089$ In CT-26-bearing mice, Juglone reduced the accumulation of MDSCs and impaired the immunosuppressive functions of MDSCs. ${ }^{112}$ In CAC mice, hyperoxia decreased G-MDSC exosome production by inhibiting HIF-1 $\alpha$-dependent Rab27a expression, which suppressed CAC susceptibility. ${ }^{12}$

Overall, MDSC infiltration is a common feature in CAC models or patients. Various strategies targeting MDSCs may be beneficial in restoring antitumor immunity in CAC and are emerging opportunities for enhancing the effectiveness of anti-CAC therapy. It is noteworthy that the experimental conditions are different in colon cancer cell-bearing mice, chemically induced CAC mice, and patients with CAC and sCRC. The characteristics of MDSCs in each organ could be different. In the bone marrow (BM) and spleen, the major role of MDSCs is immune suppression. These cells may play a protective role in IBD. MDSCs existing underneath the intestinal mucosal surface have multiple functions due to the complex intestinal microenvironment. There are close and complex relationships between MDSC characteristics and the microbiota and dietary components. Research in this area is essential for the precise treatment of CAC, and the exact effect of these factors on CAC treatments based on MDSCs remains to be further studied.

\section{CONCLUSION}

MDSCs play multiple roles in CAC initiation and progression. Cumulative evidence demonstrates that targeting MDSCs is essential for immune system reactivation in CAC. Finding combination methods to eliminate MDSCs to achieve longlasting clinical responses will be another critical issue and will be explored in future clinical trials. Due to the difficulty in manipulating human MDSCs, many of the published studies on CAC-infiltrating MDSCs have been done in mice. More studies in this area should focus on human-related content. The armamentarium for MDSC inhibition is expanding, and therefore the need to choose reasonable personalized medicine decisions will become increasingly important in the near future.

Contributors YW, YaD, and YZ contributed to the conception, content and writing. SW, YW, and YiD contributed to the revisions. All authors read and approved the final manuscript.

Funding This work was supported by the National Science Foundation of China (81902906), Research Project of Jiangsu Commission of Health (K2019019), Jiangsu Province's Key Medical Talents Program (ZDRCB2016018), Jiangsu Province's Medical Scientific Research Project (H2019102), Excellent Research Talents Cultivation Fund Program of The First People's Hospital of Yancheng City (QN2018001), and Yancheng City's Medical Science and Technology Program (YK2018004).

Competing interests None declared.

Patient consent for publication Not required.
Provenance and peer review Not commissioned; externally peer reviewed.

Open access This is an open access article distributed in accordance with the Creative Commons Attribution Non Commercial (CC BY-NC 4.0) license, which permits others to distribute, remix, adapt, build upon this work non-commercially, and license their derivative works on different terms, provided the original work is properly cited, appropriate credit is given, any changes made indicated, and the use is non-commercial. See http://creativecommons.org/licenses/by-nc/4.0/.

ORCID iD

Shengjun Wang http://orcid.org/0000-0001-6584-1183

\section{REFERENCES}

1 Low D, Mino-Kenudson M, Mizoguchi E. Recent advancement in understanding colitis-associated tumorigenesis. Inflamm Bowel Dis 2014;20:2115-23.

2 Eaden JA, Abrams KR, Mayberry JF. The risk of colorectal cancer in ulcerative colitis: a meta-analysis. Gut 2001;48:526-35.

3 Segal AW. Studies on patients establish Crohn's disease as a manifestation of impaired innate immunity. J Intern Med 2019;286:373-88.

4 Haile LA, von Wasielewski R, Gamrekelashvili J, et al. MyeloidDerived suppressor cells in inflammatory bowel disease: a new immunoregulatory pathway. Gastroenterology 2008;135:871-81.

5 Salminen A, Kaarniranta K, Kauppinen A. The role of myeloidderived suppressor cells (MDSC) in the inflammaging process. Ageing Res Rev 2018;48:1-10.

6 Elliott LA, Doherty GA, Sheahan K, et al. Human tumor-infiltrating myeloid cells: phenotypic and functional diversity. Front Immunol 2017;8:86.

7 Ostanin DV, Kurmaeva E, Furr K, et al. Acquisition of antigenpresenting functions by neutrophils isolated from mice with chronic colitis. J.i. 2012;188:1491-502.

8 Rivollier A, He J, Kole A, et al. Inflammation switches the differentiation program of Ly6Chi monocytes from antiinflammatory macrophages to inflammatory dendritic cells in the colon. $J$ Exp Med 2012;209:139-55.

9 Varol C, Vallon-Eberhard A, Elinav E, et al. Intestinal lamina propria dendritic cell subsets have different origin and functions. Immunity 2009;31:502-12.

10 Lin Q, Ren L, Jian M, et al. The mechanism of the premetastatic niche facilitating colorectal cancer liver metastasis generated from myeloid-derived suppressor cells induced by the S1PR1-STAT3 signaling pathway. Cell Death Dis 2019;10:693.

11 Wu L, Liu H, Guo H, et al. Circulating and tumor-infiltrating myeloidderived suppressor cells in cervical carcinoma patients. Oncol Lett 2018;15:9507-15.

12 Wang Y, Yin K, Tian J, et al. Granulocytic Myeloid-Derived suppressor cells promote the stemness of colorectal cancer cells through exosomal S100A9. Adv. Sci. 2019;6:1901278.

13 Bronte V, Brandau S, Chen S-H, et al. Recommendations for myeloid-derived suppressor cell Nomenclature and characterization standards. Nat Commun 2016;7:12150.

14 Dumitru CA, Moses K, Trellakis S, et al. Neutrophils and granulocytic myeloid-derived suppressor cells: immunophenotyping, cell biology and clinical relevance in human oncology. Cancer Immunol Immunother 2012;61:1155-67.

15 Huang Y-K, Wang M, Sun Y, et al. Macrophage spatial heterogeneity in gastric cancer defined by multiplex immunohistochemistry. Nat Commun 2019;10:3928.

16 Radhakrishnan J, Blechman G, Shrader C, et al. Colonic strictures following successful medical management of necrotizing enterocolitis: a prospective study evaluating early gastrointestinal contrast studies. J Pediatr Surg 1991;26:1043-6.

17 Cassetta L, Baekkevold ES, Brandau S, et al. Deciphering myeloid-derived suppressor cells: isolation and markers in humans, mice and non-human primates. Cancer Immunol Immunother 2019;68:687-97.

18 Haile LA, Gamrekelashvili J, Manns MP, et al. CD49d is a new marker for distinct myeloid-derived suppressor cell subpopulations in mice. J.i. 2010;185:203-10.

19 Rodriguez PC, Ernstoff MS, Hernandez C, et al. Arginase IProducing myeloid-derived suppressor cells in renal cell carcinoma are a subpopulation of activated granulocytes. Cancer Res 2009;69:1553-60.

20 Pillay J, Tak T, Kamp VM, et al. Immune suppression by neutrophils and granulocytic myeloid-derived suppressor cells: similarities and differences. Cell. Mol. Life Sci. 2013;70:3813-27. 
21 Arijs I, De Hertogh G, Machiels K, et al. Mucosal gene expression of cell adhesion molecules, chemokines, and chemokine receptors in patients with inflammatory bowel disease before and after infliximab treatment. Am J Gastroenterol 2011;106:748-61.

22 Dubeykovskaya Z, Si Y, Chen X, et al. Neural innervation stimulates splenic TFF2 to arrest myeloid cell expansion and cancer. Nat Commun 2016;7:10517.

23 Huang M, Wu R, Chen L, et al. S100A9 regulates MDSCs-mediated immune suppression via the RAGE and TLR4 signaling pathways in colorectal carcinoma. Front Immunol 2019;10:2243.

24 Kontaki E, Boumpas DT, Tzardi M, et al. Aberrant function of myeloid-derived suppressor cells (MDSCs) in experimental colitis and in inflammatory bowel disease (IBD) immune responses. Autoimmunity 2017;50:170-81.

25 Neut C, Bulois P, Desreumaux P, et al. Changes in the bacterial flora of the neoterminal ileum after ileocolonic resection for Crohn's disease. Am J Gastroenterol 2002;97:939-46.

26 Packey CD, Sartor RB. Interplay of commensal and pathogenic bacteria, genetic mutations, and immunoregulatory defects in the pathogenesis of inflammatory bowel diseases. J Intern Med 2008;263:597-606.

27 Hwang JH, Kim T-H, Kim Y-H, et al. Gadd $45 \beta$ promotes regeneration after injury through TGF $\beta$-dependent restitution in experimental colitis. Exp Mol Med 2019:51:1-14

28 Ostanin DV, Bhattacharya D. Myeloid-Derived suppressor cells in the inflammatory bowel diseases. Inflamm Bowel Dis 2013;19:2468-77.

29 Goldberg R, Prescott N, Lord GM, et al. The unusual suspectsinnate lymphoid cells as novel therapeutic targets in IBD. Nat Rev Gastroenterol Hepatol 2015;12:271-83.

30 Buonocore S, Ahern PP, Uhlig HH, et al. Innate lymphoid cells drive interleukin-23-dependent innate intestinal pathology. Nature 2010;464:1371-5

31 Reinisch W, de Villiers W, Bene L, et al. Fontolizumab in moderate to severe Crohn's disease: a phase 2, randomized, double-blind, placebo-controlled, multiple-dose study. Inflamm Bowel Dis 2010;16:233-42.

32 Hueber W, Sands BE, Lewitzky S, et al. Secukinumab, a human anti-IL-17A monoclonal antibody, for moderate to severe Crohn's disease: unexpected results of a randomised, double-blind placebo-controlled trial. Gut 2012;61:1693-700.

33 Geremia A, Arancibia-Cárcamo CV, Fleming MPP, et al. IL-23responsive innate lymphoid cells are increased in inflammatory bowel disease. J Exp Med 2011;208:1127-33.

34 Calcinotto A, Spataro C, Zagato E, et al. II-23 secreted by myeloid cells drives castration-resistant prostate cancer. Nature 2018;559:363-9.

35 Fallon PG, Ballantyne SJ, Mangan NE, et al. Identification of an interleukin (IL)-25-dependent cell population that provides IL-4, IL-5, and IL-13 at the onset of helminth expulsion. J Exp Med 2006;203:1105-16.

36 Tanaka J, Saga K, Kido M, et al. Proinflammatory Th2 cytokines induce production of thymic stromal lymphopoietin in human colonic epithelial cells. Dig Dis Sci 2010;55:1896-904.

37 Heller F, Florian P, Bojarski C, et al. Interleukin-13 is the key effector Th2 cytokine in ulcerative colitis that affects epithelial tight junctions, apoptosis, and cell restitution. Gastroenterology 2005;129:550-64.

38 Cao Y, He Y, Wang X, et al. Polymorphonuclear myeloid-derived suppressor cells attenuate allergic airway inflammation by negatively regulating group 2 innate lymphoid cells. Immunology 2019;156:402-12.

39 Bruce DW, Stefanski HE, Vincent BG, et al. Type 2 innate lymphoid cells treat and prevent acute gastrointestinal graft-versus-host disease. J Clin Invest 2017:127:1813-25.

40 Trabanelli S, Chevalier MF, Martinez-Usatorre A, et al. TumourDerived PGD2 and NKp30-B7H6 engagement drives an immunosuppressive ILC2-MDSC axis. Nat Commun 2017;8:593.

41 Monticelli LA, Osborne LC, Noti M, et al. II-33 promotes an innate immune pathway of intestinal tissue protection dependent on amphiregulin-EGFR interactions. Proc Natl Acad Sci U S A 2015;112:10762-7.

42 Qiu J, Zhou L. Aryl hydrocarbon receptor promotes ROR $\gamma t^{+}$group 3 ILCs and controls intestinal immunity and inflammation. Semin Immunopathol 2013;35:657-70.

43 Sartor RB. Does Mycobacterium avium subspecies paratuberculosis cause Crohn's disease? Gut 2005;54:896-8.

44 Matsuoka K, Iwao Y, Mori T, et al. Cytomegalovirus is frequently reactivated and disappears without antiviral agents in ulcerative colitis patients. Am J Gastroenterol 2007;102:331-7.
45 Darfeuille-Michaud A, Boudeau J, Bulois P, et al. High prevalence of adherent-invasive Escherichia coli associated with ileal mucosa in Crohn's disease. Gastroenterology 2004;127:412-21.

46 Nishikawa J, Kudo T, Sakata S, et al. Diversity of mucosaassociated microbiota in active and inactive ulcerative colitis. Scand $J$ Gastroenterol 2009;44:180-6.

47 Marchesi JR, Holmes E, Khan F, et al. Rapid and noninvasive metabonomic characterization of inflammatory bowel disease. $J$ Proteome Res 2007;6:546-51.

48 Jacob V, Crawford C, Cohen-Mekelburg S, et al. Single delivery of high-diversity fecal microbiota preparation by colonoscopy is safe and effective in increasing microbial diversity in active ulcerative colitis. Inflamm Bowel Dis 2017;23:903-11.

49 Thiele Orberg E, Fan H, Tam AJ, et al. The myeloid immune signature of enterotoxigenic Bacteroides fragilis-induced murine colon tumorigenesis. Mucosal Immunol 2017;10:421-33.

50 Wang T, Fan C, Yao A, et al. The adaptor protein CARD9 protects against colon cancer by restricting mycobiota-mediated expansion of myeloid-derived suppressor cells. Immunity 2018;49:504-14.

51 ZW L, Sun B, Gong T, et al. GNAl1 and GNAI3 reduce colitisassociated tumorigenesis in mice by blocking IL6 signaling and down-regulating expression of Gnai2. Gastroenterology 2019;156:2297-312.

52 Oskouian B, Sooriyakumaran P, Borowsky AD, et al. Sphingosine-1Phosphate lyase potentiates apoptosis via p53- and p38-dependent pathways and is down-regulated in colon cancer. Proc Natl Acad Sci U S A 2006;103:17384-9.

53 Ahmad S, Khan H, Siddiqui Z, et al. Ages, RAGEs and s-RAGE; friend or foe for cancer. Semin Cancer Biol 2018;49:44-55.

54 Seguella L, Capuano R, Pesce M, et al. S100B protein stimulates proliferation and angiogenic mediators release through RAGE/pAkt/ mTOR pathway in human colon adenocarcinoma Caco-2 cells. Int $J$ Mol Sci 2019;20:3240.

55 Grivennikov S, Karin E, Terzic J, et al. II-6 and STAT3 are required for survival of intestinal epithelial cells and development of colitisassociated cancer. Cancer Cell 2009;15:103-13.

56 Giles DA, Zahner S, Krause P, et al. The tumor necrosis factor superfamily members TNFSF14 (light), lymphotoxin $\beta$ and lymphotoxin $\beta$ receptor interact to regulate intestinal inflammation. Front Immunol 2018;9:2585

57 Bradford EM, Ryu SH, Singh AP, et al. Epithelial TNF receptor signaling promotes mucosal repair in inflammatory bowel disease. J.i. 2017;199:1886-97.

58 Brost S, Koschny R, Sykora J, et al. Differential expression of the TRAIL/TRAIL-receptor system in patients with inflammatory bowel disease. Pathol Res Pract 2010;206:43-50.

59 Quiros M, Nishio H, Neumann PA, et al. Macrophage-Derived IL-10 mediates mucosal repair by epithelial WISP-1 signaling. J Clin Invest 2017;127:3510-20.

60 de Barrios O, Sanchez-Moral L, Cortés M, et al. Zeb1 promotes inflammation and progression towards inflammation-driven carcinoma through repression of the DNA repair glycosylase MPG in epithelial cells. Gut 2019;68:2129-41.

61 Cen B, Lang JD, Du Y, et al. Prostaglandin E2 induces miR675$5 p$ to promote colorectal tumor metastasis via modulation of p53 expression. Gastroenterology 2020;158:971-84.

62 Wang X, Yang Y, Huycke MM. Commensal bacteria drive endogenous transformation and tumour stem cell marker expression through a bystander effect. Gut 2015;64:459-68.

63 Zhang L, Romero P. Metabolic control of CD8+ T cell fate decisions and antitumor immunity. Trends Mol Med 2018;24:30-48.

64 Kusmartsev S, Nefedova Y, Yoder D, et al. Antigen-Specific inhibition of $\mathrm{CD}^{+} \mathrm{T}$ cell response by immature myeloid cells in cancer is mediated by reactive oxygen species. J Immunol 2004;172:989-99.

65 Tian J, Rui K, Hong Y, et al. Increased GITRL impairs the function of myeloid-derived suppressor cells and exacerbates primary Sjögren syndrome. J Immunol 2019;202:1693-703.

66 Hanson EM, Clements VK, Sinha P, et al. Myeloid-Derived suppressor cells down-regulate L-selectin expression on $\mathrm{CD}^{+}$and CD8 ${ }^{+}$T cells. J Immunol 2009;183:937-44.

67 Chen J, Ye Y, Liu P, et al. Suppression of T cells by myeloid-derived suppressor cells in cancer. Hum Immunol 2017;78:113-9.

68 Deng J, Li J, Sarde A, et al. Hypoxia-Induced vista promotes the suppressive function of myeloid-derived suppressor cells in the tumor microenvironment. Cancer Immunol Res 2019;7:1079-90.

69 Noman MZ, Desantis G, Janji B, et al. Pd-L1 is a novel direct target of HIF-1 $\alpha$, and its blockade under hypoxia enhanced MDSCmediated T cell activation. J Exp Med 2014;211:781-90.

70 Kumar V, Cheng P, Condamine T, et al. Cd45 phosphatase inhibits STAT3 transcription factor activity in myeloid cells and 
promotes tumor-associated macrophage differentiation. Immunity 2016;44:303-15.

71 Toh B, Wang X, Keeble J, et al. Mesenchymal transition and dissemination of cancer cells is driven by myeloid-derived suppressor cells infiltrating the primary tumor. PLoS Biol 2011;9:e1001162.

72 Peinado $\mathrm{H}$, Zhang $\mathrm{H}$, Matei IR, et al. Pre-Metastatic niches: organspecific homes for metastases. Nat Rev Cancer 2017;17:302-17.

73 Brentnall TA, Crispin DA, Bronner MP, et al. Microsatellite instability in nonneoplastic mucosa from patients with chronic ulcerative colitis. Cancer Res 1996;56:1237-40.

74 Jernvall P, Mäkinen MJ, Karttunen TJ, et al. Microsatellite instability: impact on cancer progression in proximal and distal colorectal cancers. Eur J Cancer 1999;35:197-201.

75 Bubb VJ, Curtis LJ, Cunningham C, et al. Microsatellite instability and the role of hMSH2 in sporadic colorectalcancer. Oncogene 1996;12:2641-9.

76 Ishitsuka T, Kashiwagi H, Konishi F. Microsatellite instability in inflamed and neoplastic epithelium in ulcerative colitis. J Clin Pathol 2001;54:526-32.

77 Schulmann K, Mori Y, Croog V, et al. Molecular phenotype of inflammatory bowel disease-associated neoplasms with microsatellite instability. Gastroenterology 2005;129:74-85.

78 Smyrk TC, Watson P, Kaul K, et al. Tumor-Infiltrating lymphocytes are a marker for microsatellite instability in colorectal carcinoma. Cancer 2001;91:2417-22.

79 Llosa NJ, Cruise M, Tam A, et al. The vigorous immune microenvironment of microsatellite instable colon cancer is balanced by multiple counter-inhibitory checkpoints. Cancer Discov 2015;5:43-51.

80 Nistal E, Fernández-Fernández N, Vivas S, et al. Factors determining colorectal cancer: the role of the intestinal microbiota. Front Oncol 2015;5:220.

81 Gounaris E, Heiferman MJ, Heiferman JR, et al. Zileuton, 5-lipoxygenase inhibitor, acts as a chemopreventive agent in intestinal polyposis, by modulating polyp and systemic inflammation. PLoS One 2015;10:e0121402.

$82 \mathrm{Kim}$ YJ, Hong KS, Chung JW, et al. Prevention of colitis-associated carcinogenesis with infliximab. Cancer Prev Res 2010;3:1314-33.

83 Patlolla JM, Zhang Y, Li Q, et al. Anti-Carcinogenic properties of omeprazole against human colon cancer cells and azoxymethaneinduced colonic aberrant crypt foci formation in rats. Int $\mathrm{J}$ Oncol 2012;40:170-5

$84 \mathrm{Lu} \mathrm{Y,} \mathrm{Liu} \mathrm{H,} \mathrm{Bi} \mathrm{Y,} \mathrm{et} \mathrm{al.} \mathrm{Glucocorticoid} \mathrm{receptor} \mathrm{promotes} \mathrm{the}$ function of myeloid-derived suppressor cells by suppressing HIF1 $\alpha-$ dependent glycolysis. Cell Mol Immunol 2018;15:618-29.

85 Shi G, Li D, Ren J, et al. mTOR inhibitor INK128 attenuates dextran sodium sulfate-induced colitis by promotion of MDSCs on Treg cell expansion. J Cell Physiol 2019;234:1618-29.

86 van der Touw W, Kang K, Luan Y, et al. Glatiramer acetate enhances myeloid-derived suppressor cell function via recognition of paired Ig-like receptor B. J Immunol 2018;201:1727-34.

87 Lei A, Yang Q, Li X, et al. Atorvastatin promotes the expansion of myeloid-derived suppressor cells and attenuates murine colitis. Immunology 2016;149:432-46.

88 De Cicco P, Sanders T, Cirino G, et al. Hydrogen Sulfide Reduces Myeloid-Derived Suppressor Cell-Mediated Inflammatory Response in a Model of Helicobacter hepaticus-Induced Colitis. Front Immunol 2018;9:499.

89 Zhou J, Huang S, Wang Z, et al. Targeting EZH2 histone methyltransferase activity alleviates experimental intestina inflammation. Nat Commun 2019;10:2427.

90 Farsaci B, Higgins JP, Hodge JW. Consequence of dose scheduling of sunitinib on host immune response elements and vaccine combination therapy. Int J Cancer 2012;130:1948-59.

91 Starling N, Vázquez-Mazón F, Cunningham D, et al. A phase I study of sunitinib in combination with FOLFIRI in patients with untreated metastatic colorectal cancer. Ann Oncol 2012;23:119-27.

$92 \mathrm{Ko} \mathrm{H}$-J, Kim Y-J, Kim Y-S, et al. A combination of chemoimmunotherapies can efficiently break self-tolerance and induce antitumor immunity in a tolerogenic murine tumor model. Cancer Res 2007;67:7477-86.

93 Gonzalez-Aparicio M, Alzuguren P, Mauleon I, et al. Oxaliplatin in combination with liver-specific expression of interleukin 12 reduces the immunosuppressive microenvironment of tumours and eradicates metastatic colorectal cancer in mice. Gut 2011;60:341-9.

94 Limagne E, Euvrard R, Thibaudin M, et al. Accumulation of MDSC and Th17 cells in patients with metastatic colorectal cancer predicts the efficacy of a FOLFOX-Bevacizumab drug treatment regimen. Cancer Res 2016;76:5241-52.

95 Isambert N, Hervieu A, Rébé C, et al. Fluorouracil and bevacizumab plus anakinra for patients with metastatic colorectal cancer refractory to standard therapies (IRAFU): a single-arm phase 2 study. Oncoimmunology 2018;7:e1474319.

96 Yagiz K, Rodriguez-Aguirre ME, Lopez Espinoza F, et al. A retrovira replicating vector encoding cytosine deaminase and 5-FC induces immune memory in metastatic colorectal cancer models. Mol Ther Oncolytics 2018;8:14-26.

97 Philbrick BD, Adamson DC. Early clinical trials of Toca 511 and Toca Fc show a promising novel treatment for recurrent malignant glioma. Expert Opin Investig Drugs 2019;28:207-16.

98 Bruchard M, Mignot G, Derangère V, et al. Chemotherapy-triggered cathepsin $B$ release in myeloid-derived suppressor cells activates the NLRP3 inflammasome and promotes tumor growth. Nat Med 2013;19:57-64.

99 Belyaev NN, Abdolla N, Perfilyeva YV, et al. Daunorubicin conjugated with alpha-fetoprotein selectively eliminates myeloidderived suppressor cells (MDSCs) and inhibits experimental tumor growth. Cancer Immunol Immunother 2018;67:101-11.

100 Yuan S-J, Xu Y-H, Wang C, et al. Doxorubicin-polyglycerolnanodiamond conjugate is a cytostatic agent that evades chemoresistance and reverses cancer-induced immunosuppression in triple-negative breast cancer. J Nanobiotechnology 2019;17:110.

101 Leonard JE, Fisher TL, Winter LA, et al. Nonclinical safety evaluation of VX15/2503, a humanized IgG4 anti-SEMA4D antibody. Mol Cancer Ther 2015;14:964-72.

102 Pan P-Y, Wang GX, Yin B, et al. Reversion of immune tolerance in advanced malignancy: modulation of myeloid-derived suppressor cell development by blockade of stem-cell factor function. Blood 2008:111:219-28.

103 Liang H, Deng L, Hou Y, et al. Host STING-dependent MDSC mobilization drives extrinsic radiation resistance. Nat Commun 2017;8:8.

104 Gough MJ, Ruby CE, Redmond WL, et al. Ox40 agonist therapy enhances CD8 infiltration and decreases immune suppression in the tumor. Cancer Res 2008;68:5206-15.

105 Le DT, Uram JN, Wang $\mathrm{H}$, et al. Pd-1 blockade in tumors with mismatch-repair deficiency. N Engl J Med 2015;372:2509-20.

106 Overman MJ, McDermott R, Leach JL, et al. Nivolumab in patients with metastatic DNA mismatch repair-deficient or microsatellite instability-high colorectal cancer (CheckMate 142): an open-label, multicentre, phase 2 study. Lancet Oncol 2017;18:1182-91.

107 Lin S, Wang J, Wang L, et al. Phosphodiesterase-5 inhibition suppresses colonic inflammation-induced tumorigenesis via blocking the recruitment of MDSC. Am J Cancer Res 2017;7:41-52.

108 Nagaraj S, Youn J-I, Weber H, et al. Anti-Inflammatory triterpenoid blocks immune suppressive function of MDSCs and improves immune response in cancer. Clin Cancer Res 2010;16:1812-23.

109 Hossain F, Al-Khami AA, Wyczechowska D, et al. Inhibition of fatty acid oxidation modulates immunosuppressive functions of myeloidderived suppressor cells and enhances cancer therapies. Cancer Immunol Res 2015;3:1236-47.

110 Jayakumar A, Bothwell ALM. RIPK3-induced inflammation by IMDSCs promotes intestinal tumors. Cancer Res 2019;79:1587-99.

$111 \mathrm{Xu}$ P, Yin K, Tang X, et al. Metformin inhibits the function of granulocytic myeloid-derived suppressor cells in tumor-bearing mice. Biomed Pharmacother 2019;120:109458.

112 Wang $\mathrm{H}$, Zou C, Zhao W, et al. Juglone eliminates MDSCs accumulation and enhances antitumor immunity. Int Immunopharmacol 2019;73:118-27.

113 Youn J-I, Collazo M, Shalova IN, et al. Characterization of the nature of granulocytic myeloid-derived suppressor cells in tumor-bearing mice. J Leukoc Biol 2012;91:167-81.

114 Zhou Q, Tang X, Tian X, et al. Lncrna MALAT1 negatively regulates MDSCs in patients with lung cancer. J Cancer 2018;9:2436-42.

115 Finisguerra V, Di Conza G, Di Matteo M, et al. Met is required for the recruitment of anti-tumoural neutrophils. Nature 2015:522:349-53.

116 Manfredi AA, Ramirez GA, Rovere-Querini P, et al. The neutrophil's choice: Phagocytose vs make neutrophil extracellular traps. Front Immunol 2018:9:288.

117 Filatenkov A, Baker J, Mueller AMS, et al. Ablative tumor radiation can change the tumor immune cell microenvironment to induce durable complete remissions. Clin Cancer Res 2015;21:3727-39.

118 De Santo C, Serafini P, Marigo I, et al. Nitroaspirin corrects immune dysfunction in tumor-bearing hosts and promotes tumor eradication by cancer vaccination. Proc Natl Acad Sci U S A 2005;102:4185-90. 